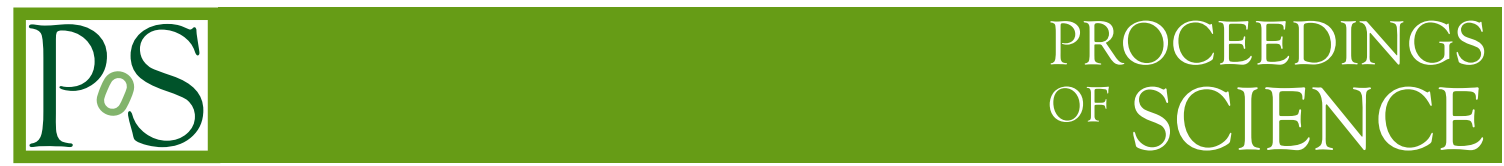

\title{
Additional neutrinos and the B anomalies
}

\section{German Valencia*i}

School of Physics and Astronomy,

Monash University,

Melbourne VIC-3800, Australia

E-mail: german.valencia@monash.edu

We discuss how additional neutrinos may help explain the B anomalies. A new light (sterile) neutrino can provide an enhancement to $R(D)$ and $R\left(D^{\star}\right)$ while satisfying existing constraints. Heavy neutrinos modify the neutral current B anomalies in the right direction, but not enough in the simplest models.

Corfu Summer Institute 2018 "School and Workshops on Elementary Particle Physics and Gravity" (CORFU2018)

31 August - 28 September, 2018

Corfu, Greece

\footnotetext{
* Speaker.

${ }^{\dagger}$ Talk based on arXiv:1711.09525 and arXiv:1706.07570.
} 


\section{One that works}

One set of B anomalies occurs in semileptonic decay modes to tau, and shows up as an apparent violation of lepton universality. The ratios $R\left(D^{(\star)}\right)=\mathscr{B}\left(\bar{B} \rightarrow D^{(\star)} \tau^{-} v\right) / \mathscr{B}\left(\bar{B} \rightarrow D^{(\star)} \ell^{-} v\right), \ell^{-}=$ $\mu^{-}, e^{-}$are measured to be larger then the standard model (SM) prediction $[1,2,3,4,5,6]$, and the combined significance of the effects is just over $4 \sigma$ [7]. The difficulty in explaining these anomalies in terms of new physics is that the charged weak current coupling to leptons has been tested very precisely (including to tau-leptons), and no violation of lepton universality is observed. This means that new physics explanations typically include a new gauge boson, charged Higgs, or leptoquark as sketched in Figure 1. The challenge any of these explanations faces is that no lepton universality

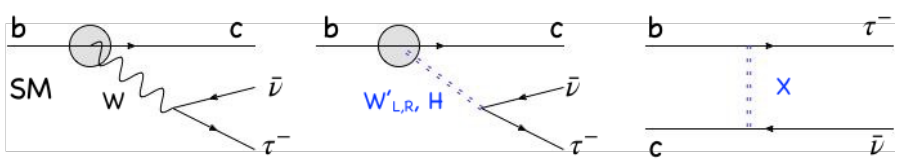

Figure 1: Schematic new particle contributions that may be responsible for the anomalies in semi-tauonic B decay. The $W \ell v$ vertex in the SM is very well constrained.

violation has been observed in other meson decay modes, some of which have been measured with much more precision than the anomalous ones. The flavor structure of the new physics must then be arranged to single out the third family, specifically b-quarks and tau-leptons.

The proposal described in this talk is built on the following ingredients

- add one new light neutrino

- this neutrino needs to be sterile with respect to SM interactions to satisfy light neutrino counts

- the neutrino needs to appear mostly in conjunction with a tau-lepton to satisfy the observed patterns of lepton flavor universality.

- one such neutrino already appears in our models that single out the third generation with a non-universal $S U(2)_{R}$

The model in which the third generation has an additional $S U(2)_{R}$ has been detailed in the literature $[8,9,10]$. The important features for the charged $\mathrm{B}$ anomalies are the existence of at least one additional neutrino, $v_{R 3}$, which has to be light in order to be relevant for this problem. It couples to the new right-handed $W_{R}$ and only through $W-W^{\prime}$ mixing to the SM $W$. The model allows for additional heavy neutrinos as well. The gauge coupling of the new $S U(2)_{R}$ can be significantly larger than the $S U(2)_{L}$ coupling $g_{L}$. There are few constraints from phenomenology for this type of $W^{\prime}$ [11], and an upper bound on the coupling strength, $g_{R} \lesssim 10 g_{L}$, arises only from requiring perturbative unitarity. In the flavor sector, the model can satisfy all known FCNC constraints, with room to accommodate deviations of electroweak strength in processes that involve a transition between a third generation fermion and lighter ones [10].

The relevant calculations for $R\left(D^{(\star)}\right)$ can be found in $[12,13]$. The results are summarized in Figure 2. There are two interesting features in this figure. First, the width of the predicted range is very thin, as it is controlled by $W-W^{\prime}$ mixing which is constrained to be tiny by $b \rightarrow s \gamma[14,12]$. 


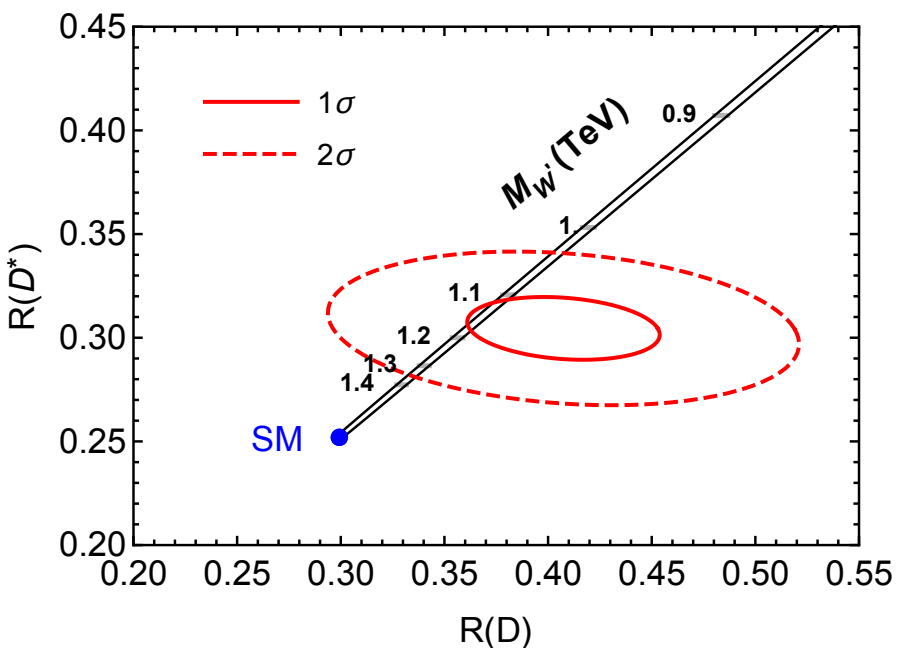

Figure 2: Results for $R\left(D^{(\star)}\right)$ as a function of $M_{W^{\prime}}$ superimposed to the $1 \sigma, 2 \sigma$ contours from HFLAV [7].

Second, the prediction is thus strongly correlated with $M_{W^{\prime}}$, and the best agreement with the fit is obtained for masses very close to $1 \mathrm{TeV}$, potentially accessible to the LHC. The best existing limit on the mass of a $W^{\prime}$ that prefers the third generation arises from a CMS search for $W^{\prime} \rightarrow \tau \nu$ with $19.7 \mathrm{fb}^{-1}$ of data at $\sqrt{s}=8 \mathrm{TeV}$ [15]. Even this constraint does not directly apply to our model, as it is extracted assuming a much larger production cross-section than the one in our model, in which the $W^{\prime}$ couples very weakly to the light fermions.

A final observation is that this model predicts an almost universal enhancement over the SM for $R\left(D^{(\star)}\right)$, as seen in Figure 2, and that this is also the same enhancement predicted for $R(J / \psi)$ [16]. Similar models that attempt to explain these anomalies with additional neutrinos have recently appeared in the literature [17, 18, 19].

Since our model relies on a new light neutrino, the light neutrino count must be addressed. Again, the direct constraint from $Z$ decay turns out to be very weak because it involves $Z-Z^{\prime}$ mixing. Our model predicts $Z \rightarrow v_{R 3} \bar{v}_{R 3}<3 \times 10^{-4} \mathrm{MeV}$, whereas the current limit on the invisible $Z$ width has an error of $13.3 \mathrm{MeV}$. At the same time, the existence of this new light neutrino affects other rare decay modes such as $K \rightarrow \pi v \bar{v}$. The current experimental results for the charged [20, 21, 22, 23] (measured by BNL 787 and BNL 949) and neutral [24] modes (from KEK E391a)are shown in Figure 3 [25]. The figure illustrates that this additional neutrino is potentially observable in these modes. In the notation of this figure $\tilde{X}$ contains the parameters of the model and it can be of order 1. An NA62 future measurement of $\mathscr{B}\left(K^{+} \rightarrow \pi^{+} v \bar{v}\right)$ at the SM level with a $30 \%$ uncertainty would place the constraint illustrated by the vertical dashed line.

An additional consideration for the viability of the model is the light neutrino count from cosmology. The BBN constraint has some uncertainty but in general requires the number of new light neutrinos to be less than about 1 . Our model evades this constraint because the new neutrino couples mostly to the third generation and effectively decouples at a temperature higher than the QCD phase transition. This means our neutrino enters this count at the 0.1 level or below. 


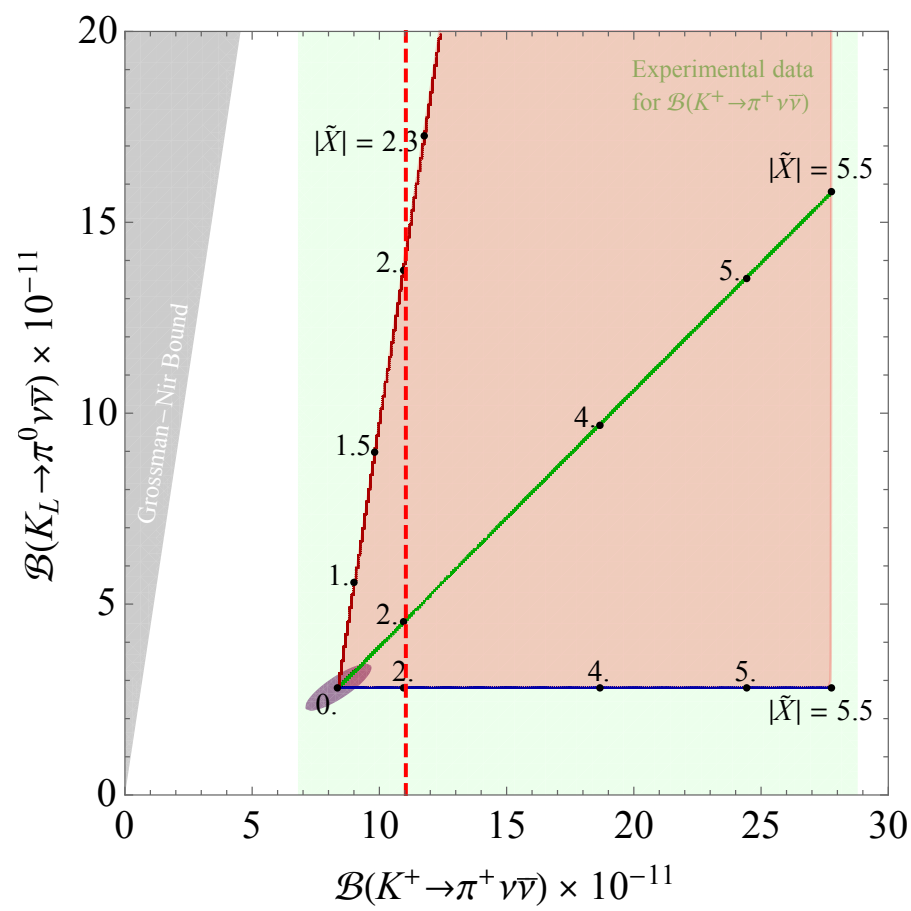

Figure 3: $K \rightarrow \pi v \bar{v}$ with one light right-handed neutrino. The green line illustrates the case $\tilde{X}$ (new physics parameters [25]) real and the pink region illustrates the case $|\tilde{X}| \leq 5.5$ (the model predicts $|\tilde{X}| \sim \mathscr{O}(1)$ ). The purple marks the SM $1 \sigma$ region and the green marks the $90 \%$ c.l. from BNL-787 combined with BNL-949. The red and blue lines on the boundary of the pink region correspond to a new physics with a certain phase. Finally the vertical dashed red line marks a possible future limit for $\mathscr{B}_{K^{+}}$at 1.3 times the SM.

\section{One that doesn't quite work}

The second set of B-anomalies occurs in modes with an underlying quark transition $b \rightarrow$ $s \mu^{+} \mu^{-}[26,27,28,29,30,31]$. Some of the largest deviations from the SM in these modes occur for the low $q^{2}$ bins of the angular observable dubbed $P_{5}^{\prime}$, and also from branching ratios in $B_{s} \rightarrow \Phi \mu \mu$. The case for lepton flavor universality violation is strongest, though, in the anomalies found in the ratios $R_{K}$ and $R_{K^{*}}$, defined as $R_{K^{(\star)}}=\mathscr{B}\left(B \rightarrow K^{(\star)} \mu^{+} \mu^{-}\right) / \mathscr{B}\left(B \rightarrow K^{(\star)} e^{+} e^{-}\right)$. The apparent shortfall of the SM becomes more compelling in the context of a global fit. For example, the authors of Ref. [32] have described 175 observables in terms of six parameters encoding new physics and have quantified the disagreement with the SM at about the $5 \sigma$ level. In Figure 5 we reproduce the result of that fit when only two new parameters are allowed to encode the new physics, $C_{9 \mu, 10 \mu}^{N P}$ [33]. The best fit is shown as the red dot and the one-sigma contour is dashed blue. In these coordinates, the SM is the origin. These two parameters are chosen because most fits indicate they are the most relevant ones.

With heavy neutrinos the SM box diagrams receive additional contributions depicted in Figure 4. Remarkably, these contributions go in the direction required by the best fit to the anomalies and appear in the form $C_{9 \mu}^{N P}=-C_{10 \mu}^{N P}$.

Early fits suggested that the pattern $C_{9 \mu}^{N P}=-C_{10 \mu}^{N P}$ was preferred by the data and this is the pattern that would be produced by heavy neutrinos. The more recent fits place this pattern outside 


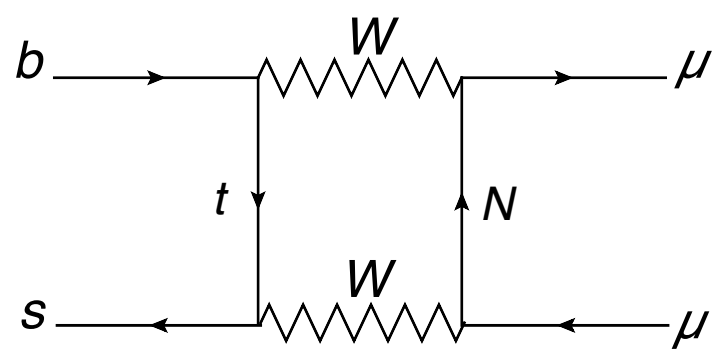

Figure 4: Heavy neutrino contributions to the anomalies in $b \rightarrow s \mu^{+} \mu^{-}$modes.

the $1 \sigma$ preferred region, but well within $2 \sigma$ as also shown in Figure 5. The right panel of the same figure shows the heavy neutrino parameters necessary to produce the needed new physics coefficients. Unfortunately, the required mixing angle between muons and the heavy neutrino is ruled out by global neutrino fits, where this mixing is at most a few percent $[34,35]$.
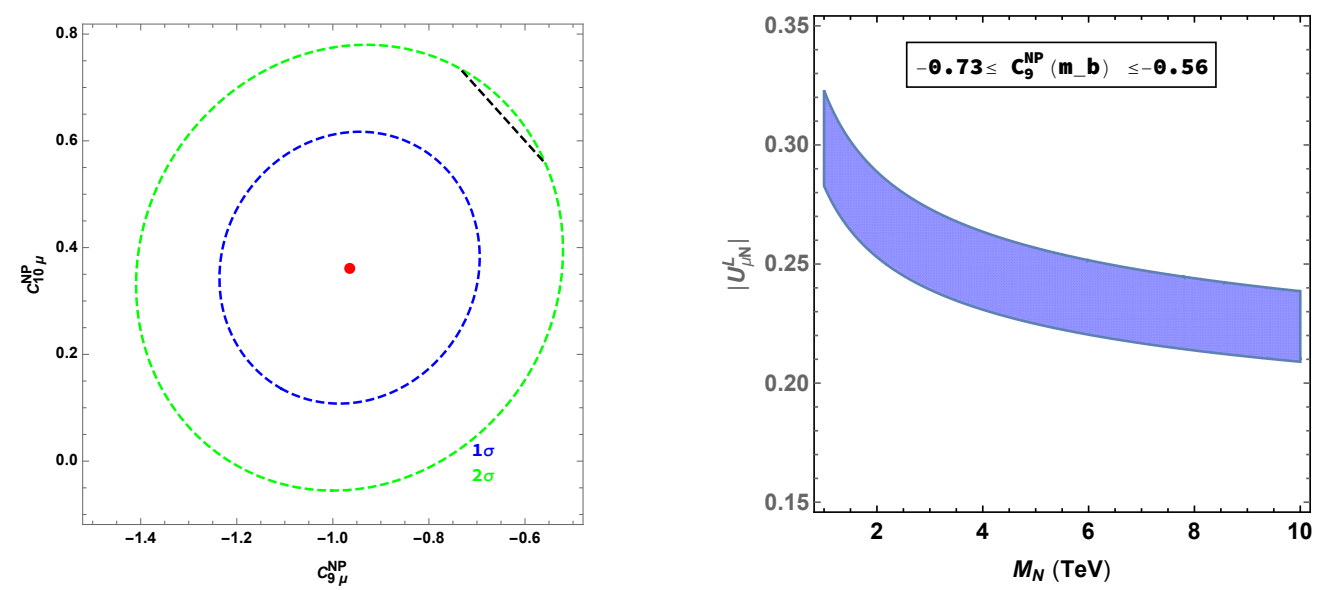

Figure 5: Left panel: Fit to 175 observables with an underlying quark transition $b \rightarrow s \mu^{+} \mu^{-}$allowing only two new parameters $C_{9 \mu, 10 \mu}^{N P}$. The red dot is the best fit, the blue and green curves mark the $1 \sigma$ and $2 \sigma$ regions respectively and the dashed black line marks $C_{9 \mu}^{N P}=-C_{10 \mu}^{N P}$ for the range $(-0.73,-0.56)$. Right panel: The region in parameter space of the heavy neutrino that reproduces the coefficients in the black line on the left.

The simplest model with heavy neutrinos does not work quantitatively, but it has been suggested in the literature that more complicated extensions could do better [36].

\section{References}

[1] J. P. Lees et al. [BaBar Collaboration], Phys. Rev. Lett. 109, 101802 (2012) doi:10.1103/PhysRevLett.109.101802 [arXiv:1205.5442 [hep-ex]].

[2] J. P. Lees et al. [BaBar Collaboration], Phys. Rev. D 88, no. 7, 072012 (2013) doi:10.1103/PhysRevD.88.072012 [arXiv:1303.0571 [hep-ex]].

[3] M. Huschle et al. [Belle Collaboration], Phys. Rev. D 92, no. 7, 072014 (2015) doi:10.1103/PhysRevD.92.072014 [arXiv:1507.03233 [hep-ex]]. 
[4] Y. Sato et al. [Belle Collaboration], Phys. Rev. D 94, no. 7, 072007 (2016) doi:10.1103/PhysRevD.94.072007 [arXiv:1607.07923 [hep-ex]].

[5] S. Hirose et al. [Belle Collaboration], Phys. Rev. Lett. 118, no. 21, 211801 (2017) doi:10.1103/PhysRevLett.118.211801 [arXiv:1612.00529 [hep-ex]].

[6] R. Aaij et al. [LHCb Collaboration], Phys. Rev. Lett. 115, no. 11, 111803 (2015) Erratum: [Phys. Rev. Lett. 115, no. 15, 159901 (2015)] doi:10.1103/PhysRevLett.115.159901, 10.1103/PhysRevLett.115.111803 [arXiv:1506.08614 [hep-ex]].

[7] http://www.slac.stanford.edu/xorg/hflav/, Y. Amhis et al., arXiv:1612.07233 [hep-ex].

[8] X. G. He and G. Valencia, Phys. Rev. D 66, 013004 (2002) [Erratum-ibid. D 66, 079901 (2002)] [arXiv:hep-ph/0203036];

[9] X. G. He and G. Valencia, Phys. Rev. D 68, 033011 (2003) [arXiv:hep-ph/0304215].

[10] X. -G. He and G. Valencia, Phys. Lett. B 680, 72 (2009) [arXiv:0907.4034 [hep-ph]].

[11] G. Valencia, Hyperfine Interact. 214, no. 1-3, 31 (2013). doi:10.1007/s10751-013-0805-x

[12] X. G. He and G. Valencia, Phys. Rev. D 87, no. 1, 014014 (2013) doi:10.1103/PhysRevD.87.014014 [arXiv:1211.0348 [hep-ph]].

[13] X. G. He and G. Valencia, doi:10.1016/j.physletb.2018.01.073 arXiv:1711.09525 [hep-ph].

[14] K. S. Babu, K. Fujikawa and A. Yamada, Phys. Lett. B 333, 196 (1994) doi:10.1016/0370-2693(94)91029-4 [hep-ph/9312315].

[15] V. Khachatryan et al. [CMS Collaboration], Phys. Lett. B 755, 196 (2016) doi:10.1016/j.physletb.2016.02.002 [arXiv:1508.04308 [hep-ex]].

[16] R. Aaij et al. [LHCb Collaboration], arXiv:1711.05623 [hep-ex].

[17] A. Greljo, D. J. Robinson, B. Shakya and J. Zupan, JHEP 1809, 169 (2018) doi:10.1007/JHEP09(2018)169 [arXiv:1804.04642 [hep-ph]].

[18] D. J. Robinson, B. Shakya and J. Zupan, JHEP 1902, 119 (2019) doi:10.1007/JHEP02(2019)119 [arXiv:1807.04753 [hep-ph]].

[19] K. S. Babu, B. Dutta and R. N. Mohapatra, JHEP 1901, 168 (2019) doi:10.1007/JHEP01(2019)168 [arXiv:1811.04496 [hep-ph]].

[20] S. Adler et al. [E787 Collaboration], Phys. Rev. Lett. 84, 3768 (2000) doi:10.1103/PhysRevLett.84.3768 [hep-ex/0002015].

[21] S. Adler et al. [E787 Collaboration], Phys. Rev. Lett. 88, 041803 (2002) doi:10.1103/PhysRevLett.88.041803 [hep-ex/0111091].

[22] V. V. Anisimovsky et al. [E949 Collaboration], Phys. Rev. Lett. 93, 031801 (2004) doi:10.1103/PhysRevLett.93.031801 [hep-ex/0403036].

[23] A. V. Artamonov et al. [BNL-E949 Collaboration], Phys. Rev. D 79, 092004 (2009) doi:10.1103/PhysRevD.79.092004 [arXiv:0903.0030 [hep-ex]].

[24] J. K. Ahn et al. [E391a Collaboration], Phys. Rev. D 81, 072004 (2010) doi:10.1103/PhysRevD.81.072004 [arXiv:0911.4789 [hep-ex]].

[25] X. G. He, G. Valencia and K. Wong, Eur. Phys. J. C 78, no. 6, 472 (2018) doi:10.1140/epjc/s10052-018-5964-0 [arXiv:1804.07449 [hep-ph]]. 
[26] LHCb Collaboration, PRL 111 (2013) 191801, arXiv:1308.1707 [hep-ex].

[27] LHCb Collaboration, JHEP 1406 (2014) 133, arXiv:1403.8044 [hep-ex].

[28] LHCb Collaboration, JHEP 1307 (2013) 084, arXiv:1305.2168 [hep-ex].

[29] LHCb Collaboration, JHEP 1504 (2015) 064, arXiv:1501.03038 [hep-ex].

[30] LHCb Collaboration, Phys. Rev. Lett. 113 (2014) 151601, arXiv:1406.6482 [hep-ex].

[31] S. Wehle et al. [Belle Collaboration], Phys. Rev. Lett. 118 (2017) no.11, 111801 [arXiv:1612.05014 [hep-ex]].

[32] B. Capdevila, A. Crivellin, S. Descotes-Genon, J. Matias and J. Virto, arXiv:1704.05340 [hep-ph].

[33] B. Capdevila, U. Laa and G. Valencia, arXiv:1811.10793 [hep-ph].

[34] S. Antusch, C. Biggio, E. Fernandez-Martinez, M. B. Gavela and J. Lopez-Pavon, JHEP 0610, 084 (2006) doi:10.1088/1126-6708/2006/10/084 [hep-ph/0607020].

[35] E. Fernandez-Martinez, J. Hernandez-Garcia and J. Lopez-Pavon, JHEP 1608, 033 (2016) doi:10.1007/JHEP08(2016)033 [arXiv:1605.08774 [hep-ph]].

[36] F. J. Botella, G. C. Branco and M. Nebot, arXiv:1712.04470 [hep-ph]. 